\title{
Reduced radial displacement of the Gastrocnemius Medialis muscle following electrically elicited fatigue
}

\begin{tabular}{|r|l|}
\hline Journal: & Journal of Sport Rehabilitation \\
\hline Manuscript ID: & JSR.2014-0325.R1 \\
\hline Manuscript Type: & Original Research Report \\
\hline Keywords: & $\begin{array}{l}\text { muscle contractile properties, maximal voluntary contraction, TMG, passive } \\
\text { muscle tension, peripheral fatigue }\end{array}$ \\
\hline Copyright Human Kinetics. This version is as accepted for publication in Journal of Sport Rehabilitation \\
\hline
\end{tabular}

\section{SCHOLARONE ${ }^{\mathrm{m}}$ \\ Manuscripts}


1 Abstract

2 Context: Assessments of skeletal muscle functional capacity often necessitate maximal

3 contractile effort, which exacerbates muscle fatigue or injury. Tensiomyography (TMG) has

4 been investigated as a means to assess muscle contractile function following fatigue;

5 however observations have not been contextualised by concurrent physiological measures.

6 Objective: The aim of the present investigation was to measure peripheral fatigue-induced

7 alterations in mechanical and contractile properties of the plantar flexor muscles through

8 non-invasive TMG concurrently with maximal voluntary contraction (MVC) and passive

9 muscle tension (PMT) in order to validate TMG as a gauge of peripheral fatigue. Design:

10 Pre- and post-test intervention with control. Setting: University laboratory. Participants:

11 Twenty-one healthy male volunteers. Interventions: Subjects plantar flexors were tested for

12 TMG parameters, along with MVC and PMT, before and after either a 5 minute rest period

13 (control) or a 5 minute electrical stimulation intervention (fatigue). Main Outcome

14 Measures: Temporal (contraction velocity) and spatial (radial displacement) contractile

15 parameters of the Gastrocnemius Medialis were recorded through TMG. MVC was

16 measured as an indicator of muscle fatigue and PMT was measured to assess muscle

17 stiffness. Results: Radial displacement demonstrated a fatigue-associated reduction (3.3 \pm

181.2 vs. $4.0 \pm 1.4 \mathrm{~mm}$ vs, $p=0.031$ ), while contraction velocity remained unaltered.

19 Additionally, MVC significantly declined by $122.6 \pm 104 \mathrm{~N}(\mathrm{p}<0.001)$ following stimulation

20 (fatigue). PMT was significantly increased following fatigue (139.8 \pm 54.3 vs. $111.3 \pm 44.6$

$21 \mathrm{~N}, \mathrm{p}=0.007$ ). Conclusion: TMG successfully detected fatigue, evident from reduced MVC,

22 by displaying impaired muscle displacement, accompanied by elevated PMT. TMG could 
23 be useful in establishing fatigue status of skeletal muscle without exacerbating the

24 functional decrement of the muscle.

26 Key words: muscle contractile properties, maximal voluntary contraction, TMG, passive 27 muscle tension, peripheral fatigue

\section{Introduction}

Muscle fatigue is characterised by a decrease in the external force or torque generating capacity, ${ }^{1}$ and/or by impairment in peak power output. ${ }^{2}$ The manifestation and magnitude of this reduced function depends upon multiple factors including the muscle contraction mode, ${ }^{1}$ the nature of the fatigue protocol ${ }^{3}$ and the source of the fatigue. ${ }^{4}$ Fatigue-related alterations of skeletal muscle can be observed, amongst other factors, by changes in its contractile and mechanical properties.

Since fatigue is a condition that affects both athletic performance and clinical mobility, the need for a valid monitor of muscle response is important to enable optimal management of athletes and patients. In situations of muscle fatigue, or indeed musculoskeletal injury, it is impractical to assess muscle function through a measure which makes use of voluntary efforts (i.e. MVC), due to centrally mediated inhibition. ${ }^{5}$ Furthermore, the potential for aggravation of any damage to the musculoskeletal unit cannot be ruled out. Having been developed over the last 15 years, tensiomyography (TMG) is a portable and non-invasive means of measuring muscle response through combined use of sub-maximal (below 
45 to that used in mechanomyography. ${ }^{9}$ TMG records spatial and temporal parameters of the 46 radial displacement of the muscle belly in response to electrical stimuli ${ }^{10}$ and is reliable 47 within ${ }^{11}$ and between days. ${ }^{12}$ Furthermore, TMG has also demonstrated good long-term 48 stability following fatigue ${ }^{13}$ and has displayed significant interclass correlation coefficient 49 with decline and recovery of maximal voluntary contraction (MVC) following exercise50 induced muscle damage. ${ }^{14}$ In particular muscle displacement (Dm) and contraction time 51 (Tc) have shown greatest stability. ${ }^{12}$

52 TMG has successfully detected fatigue-associated changes following ultra-endurance 53 triathlon, ${ }^{15}$ and resistance exercise. ${ }^{16}$ However, these studies report inconsistent results in 54 the fatigue-induced alteration of the TMG parameters, perhaps due to the vast differences 55 in the fatigue protocols administered and the different muscles measured. Furthermore, previous studies have failed to relate the TMG alterations to any valid functional measure,

57 such as maximal voluntary contraction (MVC) or passive muscle tension (PMT), which leaves the physiological interpretation of the TMG data open to question. Therefore, in order to effectively provide meaningful validation of TMG measurement to local fatigue it is 60 important to overcome this limitation. In practical terms, sub-maximal TMG could offer an 61 attractive measure for sport and medical practitioners in their assessment of muscle 62 response and status following fatigue based activity without necessitating voluntary 63 contractile effort.

Accordingly, the aim of the present investigation was to evaluate peripheral fatigue-induced alterations in mechanical and contractile properties of the Gastrocnemius muscle, as measured by TMG. MVC and PMT were measured before and after intervention, to quantify

67 the extent of muscle fatigue, and allow us to better interpret changes in TMG response; to 
68 our knowledge this has not been previously reported. It was hypothesised that a reduction

69 in size and velocity of muscle displacement would indicate muscle fatigue in line with 70 impairments in muscle function (decreased MVC) and elevated muscle stiffness (increased 71 PMT). The findings of this study could help to establish TMG as a non-invasive alternative 72 to quantify muscle fatigue.

\section{Methods}

Participants

76 Twenty-one healthy males with a mean $( \pm$ SD) age, height, and mass of $21.3 \pm 3.4$ years, $182.0 \pm 6.1 \mathrm{~cm}$, and $79.5 \pm 10.0 \mathrm{~kg}$, volunteered and gave their written informed consent to participate in this study. All participants were recreationally active and free from injury. Females were excluded from the study in order to maintain cohort homogeneity. The study was performed in accordance with the principles outlined in the Declaration of Helsinki and was approved by the local research ethics committee.

Design

Mechanical and contractile properties of the right Gastrocnemius Medialis (GM) were monitored using TMG (BMC Ltd, Ljubljana). GM is one of the propulsive muscles, fundamental to different types of human locomotion and is located superficially, making it clearly measurable by TMG. Participants were also tested for PMT and MVC of the right plantar flexors. Testing was carried out on two occasions, one week apart, as illustrated in figure 1. Measurements were taken at a number of time points before and after either the control or fatigue intervention, according to the following order: TMG and PMT 
90 (measurement 1, M1), warm-up, TMG and PMT + MVC (M2), either control or fatigue 91 intervention in random order, TMG and PMT + MVC (M3). Both TMG and PMT 92 measurements were recorded three minutes after the warm-up, and after the control or 93 fatigue intervention, to limit the effects of post activation potentiation in the GM muscle. ${ }^{18}$

94 Participants reported to the laboratory on the morning of each experimental trial in a fasted and rested state. Twenty-four hour dietary intake records were completed on the day preceding each trial, and participants were instructed to replicate their dietary intake before each visit.

Warm-up

Participants warmed up by cycling at a low intensity (75 Watts) on an electromagnetically braked cycle ergometer (Lode Ergometer, Netherlands) for 5 minutes at a cadence between 80 and $90 \mathrm{rpm}$.

TMG measurements were performed exactly as described by Ditroilo et al (2013). ${ }^{13}$ Briefly, participants lay in a prone position on a padded bench. A foam pad, placed slightly proximal to the ankle joint, supported a knee flexion angle of around $5^{\circ}$. The digital displacement transducer (TMG-BMC Ltd, Ljubljana) was then positioned perpendicular to consistently retracting the spring-loaded transducer probe to $50 \%$ of its length. This measuring position was selected by first manually palpating the GM to locate the thickest 
112 highest mechanical response with the least amount of co-activation when externally

113 stimulated; co-activation was typically identified by a second peak in the TMG response

114 curve. Once the appropriate position was obtained, it was marked with a permanent marker

115 pen to ensure exact uniformity when the sensor was repositioned for subsequent

116 measurements. The centre point of each of the 2 stimulating electrodes $\left(5 \mathrm{~cm}^{2}\right)$ (Axelgaard,

117 USA) was located approximately half way from the position of the sensor $(\sim 5 \mathrm{~cm})$ to the start

118 of the respective GM proximal distal tendons. After each measurement these electrodes

119 were left in place and unplugged to avoid any possible changes in muscle response via

120 alterations in surface electrodes distance. ${ }^{10} \mathrm{~A}$ single $1 \mathrm{~ms}$ wide stimulation pulse was

121 delivered, which applied initial current amplitude of $20 \mathrm{~mA}$. This amplitude was

122 progressively increased by $10 \mathrm{~mA}$ increments until maximal response was obtained, i.e. no

123 further displacement of the muscle belly could be produced as identified by a plateau in

124 the twitch response curves. In order to minimize the effects of fatigue and potentiation,

125 rest periods of 10 seconds were allowed between each stimulation pulse. Typical maximal

126 responses were observed at amplitude between 40 and $70 \mathrm{~mA}$ and only the output data for

127 that particular stimulation intensity were used for analysis. Figure 2 shows a typical TMG

128 displacement/ time curve before and after the administration of the fatigue protocol.

129 Output parameters were extracted and analysed from each maximal twitch response: ${ }^{10}$

130 Displacement $(\mathrm{Dm})$, the extent of maximal radial deformation $(\mathrm{mm})$ of the muscle belly

131 during contraction; Contraction velocity $(\mathrm{Vc})$, the rate $\left(\mathrm{mm}^{-1}\right)$ of contraction between $10 \%$

132 and $90 \%$ of maximal displacement. Raw data were extracted from the TMG software and

133 Vc was calculated according to the formula: $[\mathrm{Vc}=\mathrm{Dm} 80 / \mathrm{Tc}]$ where $\mathrm{Tc}=$ contraction time

134 between $10 \%$ and $90 \%$ of peak radial displacement of the muscle belly; Dm80 = the radial

135 displacement occurring during the time period of Tc. ${ }^{19}$ Muscle contraction time (Tc) has 
136 been widely reported in previous studies, ${ }^{10,15-16}$ as the temporal change from $10 \%-90 \%$ of 137 muscle Dm, providing a value relative to the spatial characteristics of each muscle. However, 138 when assessing intramuscular alterations, i.e. pre- and post- fatigue, the significance of 139 calculating Tc in this manner should be questioned. Indeed, in the absence of signal 140 latency, it is possible that a decrease in Dm could associate with a decrease in Tc, when 141 calculated as described above. Apparent decreases in Tc, suggesting a faster twitch

Maximal voluntary contraction (MVC) protocol

Plantar flexor isometric MVC was performed in an isokinetic dynamometer (Kin-Com, Chattanooga Group Inc., USA). The participant had their right foot fastened securely into

Measurements of PMT of the right plantar flexors were made on the same isokinetic 157 dynamometer, with a set-up identical to the MVC protocol (figure 3). Participants were 
158 instructed to completely relax once in position, and the mean passive force of the ankle flexed at $90^{\circ}$ was recorded during a period of $15 \mathrm{~s}$, as a measure of passive muscle tension

160 in the plantar flexors in a static position. ${ }^{20} \mathrm{~A}$ single measure was taken to determine PMT, as subsequent stretching of the ankle joint would cause an accumulative stretch effect. An intra-session reliability, as measured by the intraclass correlation coefficient, $\geq 0.80$ has been previously reported for this type of measurement. ${ }^{21}$

The fatigue intervention used in the current investigation differs from previous studies in this area ${ }^{15,16}$ in a number of key ways. Firstly, fatigue was induced locally with a low frequency stimulation that will necessitate a prolonged recovery, compared to higher frequency fatigue. ${ }^{17}$ Secondly, as motor unit discharge rarely exceeds $30 \mathrm{~Hz}$ during voluntary contraction, ${ }^{17}$ low frequency stimulus can be considered a more functionally relevant intervention. Finally, as TMG is a passive and peripheral measurement it will minimise confounding variables such as the variability of central control factors. Whilst remaining secured in the same position as for PMT the participants received the fatigue intervention, which consisted of a 5 minute electrical stimulation of the right $G M$, to evoke fatigue. The stimulation protocol involved a train of 15 electrical pulses ( 1 every $100 \mathrm{~ms}$ ) with a 1 second gap before the start of each subsequent train. The protocol lasted 5 minutes and participants were asked to endure the maximum current they could, to ensure fatigue ( 110 mA). The control intervention consisted of the same positioning but receiving no 178 stimulation for a period of 5 minutes to account for the effect of time. Also in the same position, with the ankle placed at $90^{\circ}$, isometric MVC of the plantar flexors was measured, before and after both intervention and control, to assess whether fatigue occurred. Each 
181 participant performed three 5 second MVCs, with 60 seconds recovery between attempts.

182 Participants were provided with consistent verbal motivation to ensure maximal effort 183 throughout.

Statistical Analysis

All data are presented as mean \pm SD. After testing for assumption of normality of the dependent variables and log-transforming where necessary (i.e. when not normally distributed), a 3 (measurements: before warm-up, M1; after warm-up, M2; after intervention, M3) $\times 2$ (condition: control and fatigue intervention) ANOVA with repeated measures on both factors was used to detect differences in PMT and TMG parameters as a result of the fatigue/ control protocol. Where a significant F value was found a Tukey post hoc test was used to identify where any significant difference occurred. Paired $t$-test was conducted to compare the pre- / post-fatigue MVC difference between the control and fatigue intervention. Effect size (ES) was also calculated using eta-squared (n2) and 194 interpreted as small (0.01), moderate (0.06) or large $(0.14) .^{22}$ The percentage differences between control and fatigue intervention were also calculated and interpreted based on the 196 minimum detectable change as reported in a previous reliability study. ${ }^{13}$ An alpha level of $p$ $197<0.05$ was considered statistically significant. Statistical analysis was performed using 198 Statistica version 10 (Statsoft LTD, Bedford, UK).

Results 
202 Dm demonstrated a fatigue-associated alteration. A significant main effect for 'condition'

$203\left(F=7.2, p=0.002, \eta^{2}=0.27\right)$ was documented for $\mathrm{Dm}$, along with a post-hoc difference at 204 M3 demonstrating that the fatigue condition was significantly lower than control 205 condition $(3.3 \pm 1.2$ vs $4.0 \pm 1.4 \mathrm{~mm}, p=0.031$; figure 4$)$, with a percentage difference of 206 17.7\%. No significant difference was found for any of the factors or their interaction for 207 Vc, which exhibited $121.8 \pm 43.2$ vs $124.7 \pm 45.5 \mathrm{~mm}^{-1}$ at $\mathrm{M} 1,121.3 \pm 45.7$ vs $124.9 \pm 44.7$ $208 \mathrm{~mm}^{-1}$ at M2, $131.3 \pm 44.6$ vs $139.8 \pm 50.6 \mathrm{~mm}^{-1}$ at $\mathrm{M} 3$.

210 Plantar flexor isometric MVC exhibited a significant interaction 'condition $\mathbf{x}$ measurement' $211\left(F=12.4, p=0.001, \eta^{2}=0.91\right)$ with post-hoc analysis showing a significant decline following 212 the fatigue intervention $(-122.6 \pm 104 \mathrm{~N} ; \mathrm{p}<0.001)$ but not following control $(-25.7 \pm 71.3$ $213 \mathrm{~N}, \mathrm{p}=\mathbf{0 . 1 1 5})$. The PMT exhibited a significant interaction 'condition $\mathrm{x}$ measurement' ( $\mathrm{F}=5.9$, $214 p=0.005, \eta^{2}=0.23$ ). The post-hoc analysis revealed at M3 that fatigue caused significantly 215 more tension than control $(139.8 \pm 54.3$ vs. $111.3 \pm 44.6 \mathrm{~N}, \mathrm{p}=0.007$; figure 5$)$, with a 216 percentage difference of $\mathbf{2 0 . 4 \%}$.

Discussion

This study was designed to evaluate the validity of TMG, as a sub-maximal assessment method, to detect local muscular fatigue, against functional physiological measures. Fatigue of the GM was achieved, as evidenced by the significant decline in peak force (MVC), which was absent following the control condition. This alteration in functional capacity of the 
224 following dynamic fatigue. ${ }^{16,23}$ In addition, plantar flexor PMT increased following the 225 fatigue intervention suggesting that the GM skeletal muscle-tendon unit became stiffer. 226 Despite these alterations, muscle twitch Vc appeared to remain unaffected by fatigue.

227 When considering the physiological effects of fatigue there are a number of important variables to examine. We have previously demonstrated that during fatigued voluntary contractions muscle fibre conduction velocity declines due to a reduction in extracellular

$\mathrm{pH}^{24}$ It is likely that this occurs due to a $\mathrm{pH}$ driven alteration of the $\mathrm{Na}+$ and $\mathrm{K}+$ gradient across the sarcolemma ${ }^{25}$ and impairs action potential propagation. Therefore, during TMG measurement the electrical stimulus applied to the surface of the fatigued muscle should result in a slowing down of the action potentials propagated to reduce Ca2+ release and subsequent excitation-contraction (E-C) coupling. Low-frequency fatigue, as characterized by a disproportionate reduction in force at lower stimulation frequencies, has been associated with $\mathrm{E}-\mathrm{C}$ uncoupling. ${ }^{26}$ It has been suggested that $\mathrm{E}-\mathrm{C}$ uncoupling is attributable to, amongst other factors, impaired Ca2+ transport via Ryanodine receptor channels in the triadic compartment. ${ }^{27}$ Furthermore, other contributing factors will be from increased $\mathrm{Pi}$ which can push the cross-bridge into a low force generating status ${ }^{28}$ and may also cause actin and myosin to detach. ${ }^{29}$ These altered characteristics of muscle function will inevitably impair its force generation capacity, as shown by the significant decline in MVC.

It has been reported previously that a stiffer muscle, as we have evidenced here by the rise in PMT (figure 5), will be associated with a reduced TMG Dm measurement. ${ }^{8}$ In contrast to the current findings, Garcia-Manso et $\mathrm{al}^{15}$ showed an increase in Biceps Femoris TMG Dm associated with fatigue following an ironman triathlon. The precise 
247 showed a small decline in whole leg stiffness during a running task, following a 24-hour

248

249

250

251

252

253

254

255

256

257

258

259

260

261

262

263

264

265

266

267

268

269

marathon. These authors postulated that central fatigue would have been apparent which would have been linked to altered peripheral feedback from muscle afferents triggered from cytokines. This, we suggest, may be why an increase in TMG Dm was observed following an ironman triathlon when a decline has been reported with other types of fatigue from far shorter contractile/ exercise durations. Other studies have also demonstrated alterations in Dm alongside muscle architectural changes. Firstly, Pisot et al, ${ }^{8}$ showed that following 35 days of bed rest, TMG Dm increased alongside the reduction in muscle thickness which the authors suggested would have contributed to reduced muscle stiffness. Secondly, we previously demonstrated ${ }^{31}$ that altering the length of the muscle will determine the magnitude of TMG parameters, such that longer muscle length, as achieved through altered joint angle, results in reduced Dm. Thirdly, although not relating the decline in TMG Dm to muscle stiffness changes, other studies ${ }^{16,23}$ have also demonstrated a decline in TMG Dm following fatigue, suggesting that this is an important parameter when assessing the muscle status in this regard.

In the present study we observed decreases in TMG Dm without significant alterations in Vc. Given previously described reductions in action potential propagation and muscle fibre conduction velocity, associated with fatigue, ${ }^{24}$ it may have been expected that TMG Vc would be observed to decline post-fatigue, in concurrence with $\mathrm{Dm}$. It is plausible that the lack of significant alteration in $\mathrm{Vc}_{\mathrm{c}}$ is due to the high degree of inter-individual variability associated with the measurement. Indeed, changes between measurements (M1, M2, M3) ranged from about $-25 \%$ to $+25 \%$ between participants. The comparably low amplitude of the electrical stimulation used to elicit the peak TMG response, may perhaps render these 
data difficult to compare to existing conduction velocity findings. As such, it may be inappropriate to consider alterations in the speed/ time component of the TMG response,

272 when assessing muscle fatigue, with the focus instead being placed on spatial alterations

$273(\mathrm{Dm})$, which we have shown here to be indicative of increased muscle stiffness.

As with any type of physiological measurement there will be a degree of variability. We have previously accounted for this variability with TMG measured under different muscle conditions $^{13}$ and shown $\mathrm{Dm}$ to be well within acceptable limits. Analogous to this is establishing minimal detectable change so practitioners and researchers can be confident that the given magnitude of observed change following any intervention is real and physiologically significant. We have demonstrated in this study that the fatigue-altered Dm parameter $(17.7 \%)$ clearly exceeds the minimal detectable change thresholds of $15.1 \% .{ }^{13}$ Furthermore, the effect size for the data presented in this study, as described by Cohen, ${ }^{22}$ is "large" suggesting that this particular TMG measure is sufficiently sensitive to adequately detect local muscular fatigue. Nonetheless, a number of limitations must be considered. Current findings can only be applied to a healthy, young male population. It remains to be seen whether TMG measurements are sufficiently sensitive to detect fatigue associated changes in alternative cohorts. Additionally, GM was selected for investigation as its anatomical position facilitates measurement using TMG. Muscles which are not located superficially, but may still be of interest, are not measureable using the methods described herein.

\section{Conclusion}

This is the first study to demonstrate that TMG was effective in detecting local muscular fatigue in the GM. We propose that this response was directly related to increased stiffness 
293 of the muscle from impaired contractile capacity. It should be emphasised that, when 294 assessing local muscular fatigue, Dm of the muscle is a valid measure, however it remains 295 to be seen whether TMG has the sensitivity to detect any changes in Vc in a different 296 context. The current findings have important implications for researchers and practitioners 297 seeking to establish fatigue status of skeletal muscle, with implications for prevention of 298 over-training injuries in sports-related activities. Given the non-invasive and sub-maximal nature of this type of measurement, TMG can be used to determine local muscular fatigue 300 in patients who may be unable to exert the maximal effort required for voluntary muscle 301 function assessments. Additionally, TMG measurements are exempt from the bias of volitional effort and motivation, facilitating the incorporation of the procedure into existing programmes. ${ }^{32}$ Furthermore, TMG could be utilised regularly, as a monitoring tool, without fear of detriment to muscle function.

References

1. Garrandes, F., Colson, S. S., Pensini, M., Seynnes, O., \& Legros, P. Neuromuscular fatigue profile in endurance-trained and power-trained athletes. Medicine and Science in Sports and

311 Exercise, 2007; 39 (1): 149-158.

2. Ditroilo, M., Watsford, M., Fernandez-Pena, E., D’Amen, G., Lucertini, F., \& De Vito, G.

313 Effects of fatigue on muscle stiffness and intermittent sprinting during cycling. Medicine \& 314 Science in Sports \& Exercise, 2011; 43 (5): 837-845. 
315 3. Babault, N., Desbrosses, K., Fabre, M.-S., Michaut, A., \& Pousson, M. Neuromuscular 316 fatigue development during maximal concentric and isometric knee extensions. Journal of 317 Applied Physiology, 2006; 100 (3): 780-785.

4. Berchicci, M., Menotti, F., Macaluso, A. \& Di Russo, F. The neurophysiology of central and peripheral fatigue during sub-maximal lower limb isometric contractions. Frontiers in Human Neuroscience, 2013; 7 (135).

5. Graven-Nielsen, T., Lund, H., Arendt-Nielsen, L., Danneskiold-Samsøe, B., \& Bliddal, H. Inhibition of maximal voluntary contraction force by experimental muscle pain: a centrally mediated mechanism. Muscle \& nerve, 2002; 26(5): 708-712.

6. Dahmane, R., Valenčič, V., Knez, N., \& Erzen, I. Evaluation of the ability to make noninvasive estimation of muscle contractile properties on the basis of the muscle belly response. Medical \& Biological Engineering \& Computing, 2001; 39: 51-55.

7. Dahmane, R., Djordjevic, S., Simunič, B., Valenčič, V. Spatial fiber type distribution in normal human muscle Histochemical and tensiomyographical evaluation. Journal of Biomechanics, 2005; 38: 2451-2459.

8. Pisot, R., Narici, M. V, Simunič, B., De Boer, M., Seynnes, O., Jurdana, M., Biolo, G., \& Mekjavić, I. B. Whole muscle contractile parameters and thickness loss during 35-day bed rest. European Journal of Applied Physiology, 2008; 104 (2): 409-414. Sons; 2004. 
336 10. Tous-Fajardo, J., Moras, G., Rodriguez-Jimenez, S., Usach, R., Doutres, D. M., \&

337 Maffiuletti, N. A. Inter-rater reliability of muscle contractile property measurements using 338 non-invasive tensiomyography. Journal of Electromyography and Kinesiology, 2010; 20 (4): $339761-766$.

340 11. Krizaj, D., Simunič, B., \& Zagar, T. Short-term repeatability of parameters extracted from 341 radial displacement of muscle belly. Journal of Electromyography and Kinesiology, 2008; 18 342 (4): 645-651.

343 12. Simunič, B. Between-day reliability of a method for non-invasive estimation of muscle 344 composition. Journal of Electromyography and Kinesiology, 2012; 22 (4): 527-530.

345 13. Ditroilo, M., Smith, I. J., Fairweather, M. M., \& Hunter, A. M. Long-term stability of 346 tensiomyography measured under different muscle conditions. Journal of Electromyography 347 and Kinesiology, 2013; 23 (3): 558-563.

348 14. Hunter, A. M., Galloway, S. D. R., Smith, I. J., Tallent, J., Ditroilo, M., Fairweather, M. M., 349 \& Howatson, G. Assessment of eccentric exercise-induced muscle damage of the elbow 350 flexors by tensiomyography. Journal of Electromyography and Kinesiology, 2012; 22 (3): $351 \quad 334-341$. 15. Garcia-Manso, J. M., Rodriguez-Ruiz, D., Rodriguez-Matoso, D., de Saa, Y., Sarmiento, S., \& Quiroga, M. Assessment of muscle fatigue after an ultra-endurance triathlon using 354 tensiomyography (TMG). Journal of Sports Sciences, 2011; 29 (6): 619-625.

16. Garcia-Manso, J. M., Rodriguez-Matoso, D., Sarmiento, S., de Saa, Y., Vaamonde, D., 356 Rodriguez-Ruiz, D., \& Da Silva-Grigoletto, M. E. Effect of high-load and high-volume 
357 resistance exercise on the tensiomyographic twitch response of biceps brachii. Journal of 358 Electromyography and Kinesiology, 2012; 22 (4): 612-619.

359 17. Allen, D. G., Lamb, G. D., \& Westerblad, H. Skeletal muscle fatigue: cellular mechanisms. 360 Physiological Reviews, 2008; 88 (1): 287-332.

18. Paasuke, M., Saapar, L., Ereline, J., Gapeyeva, H., Requena, B., \& Oopik, V. Postactivation 362 potentiation of knee extensor muscles in power- and endurance-trained, and untrained 363 women. European Journal of Applied Physiology, 2007; 101 (5): 577-585.

19. Valenčič, V., \& Knez, N. Measuring of skeletal muscles' dynamic properties. Artificial 365 Organs, 1997; 21 (3): 240-242.

20. Fowles, J. R., Sale, D. G., \& MacDougall, J. D. Reduced strength after passive stretch of 367 the human plantarflexors. Journal of Applied Physiology, 2000; 89 (3): 1179-1188.

21. Araújo, V. L., Carvalhais, V. O. C., Souza, T. R., Ocarino, J. M., Gonçalves, G. G. P., \&

Fonseca, S. T. Validity and reliability of clinical tests for assessing passive ankle stiffness. Revista Brasileira de Fisioterapia, 2011; 15 (2): 166-173.

22. Cohen, J. Statistical Power Analysis for the Behavioural Sciences (2nd ed.). Hillsdale, NJ: Lawrence Erlbaum Associates; 1988.

23. Carrasco, L., Sanudo, B., de Hoyo, M., Pradas, F., \& Da Silva, M. E. Effectiveness of low374 frequency vibration recovery method on blood lactate removal, muscle contractile 375 properties and on time to exhaustion during cycling at $\mathrm{VO}_{2}$ max power output. European 376 Journal of Applied Physiology, 2011; 111 (9): 2271-2279. 
377 24. Hunter, A. M., De Vito, G., Bolger, C., Mullany, H., \& Galloway, S. D. R. The effect of 378 induced alkalosis and submaximal cycling on neuromuscular response during sustained 379 isometric contraction. Journal of Sports Sciences, 2009; 27 (12): 1261-1269.

380 25. Brody, L. R., Pollock, M. T., Roy, S. H., De Luca, C. J., \& Celli, B. pH-induced effects on 381 median frequency and conduction velocity of the myoelectric signal. Journal of Applied 382 Physiology, 1991; 71 (5): 1878-1885.

26. Hill, C. A., Thompson, M. W., Ruell, P. A., Thom, J. M. \& White, M. J. Sarcoplasmic 384 reticulum function and muscle contractile character following fatiguing exercise in humans. 385 Journal of Physiology, 2001; 531: 871-878.

27. Balog, E. M. Excitation-contraction coupling and minor triadic proteins in low-frequency fatigue. Exercise and Sport Sciences Reviews, 2010; 38 (3): 135-142.

28. Nocella, M., Colombini, B., Benelli, G., Cecchi, G., Bagni, M. A., \& Bruton, J. Force decline during fatigue is due to both a decrease in the force per individual cross-bridge and the number of cross-bridges. The Journal of Physiology, 2011; 589 (Pt 13): 3371-3381.

29. Takagi, Y., Shuman, H., \& Goldman, Y. E. Coupling between phosphate release and force generation in muscle actomyosin. Philosophical Transactions of the Royal Society of London. Series B, Biological Sciences, 2004; 359 (1452): 1913-1920.

30. Morin, J. B., Tomazin, K., Edouard, P., \& Millet, G. Y. Changes in running mechanics and spring-mass behaviour induced by a mountain ultra-marathon race. Journal of Biomechanics, 2011; 44 (6): 1104-1107. 
397 31. Ditroilo, M., Hunter, A. M., Haslam, S., \& De Vito, G. The effectiveness of two novel 398 techniques in establishing the mechanical and contractile responses of biceps femoris. 399 Physiological Measurement, 2011; 32 (8): 1315-1326.

400 32. Rey, E., Lago-Peñas, C., \& Lago-Ballesteros, J. Tensiomyography of selected lower-limb 401 muscles in professional soccer players. Journal of Electromyography and Kinesiology, 2012;

$402 \quad 22(6): 866-872$. 
Testing Session 1

Testing Session 2
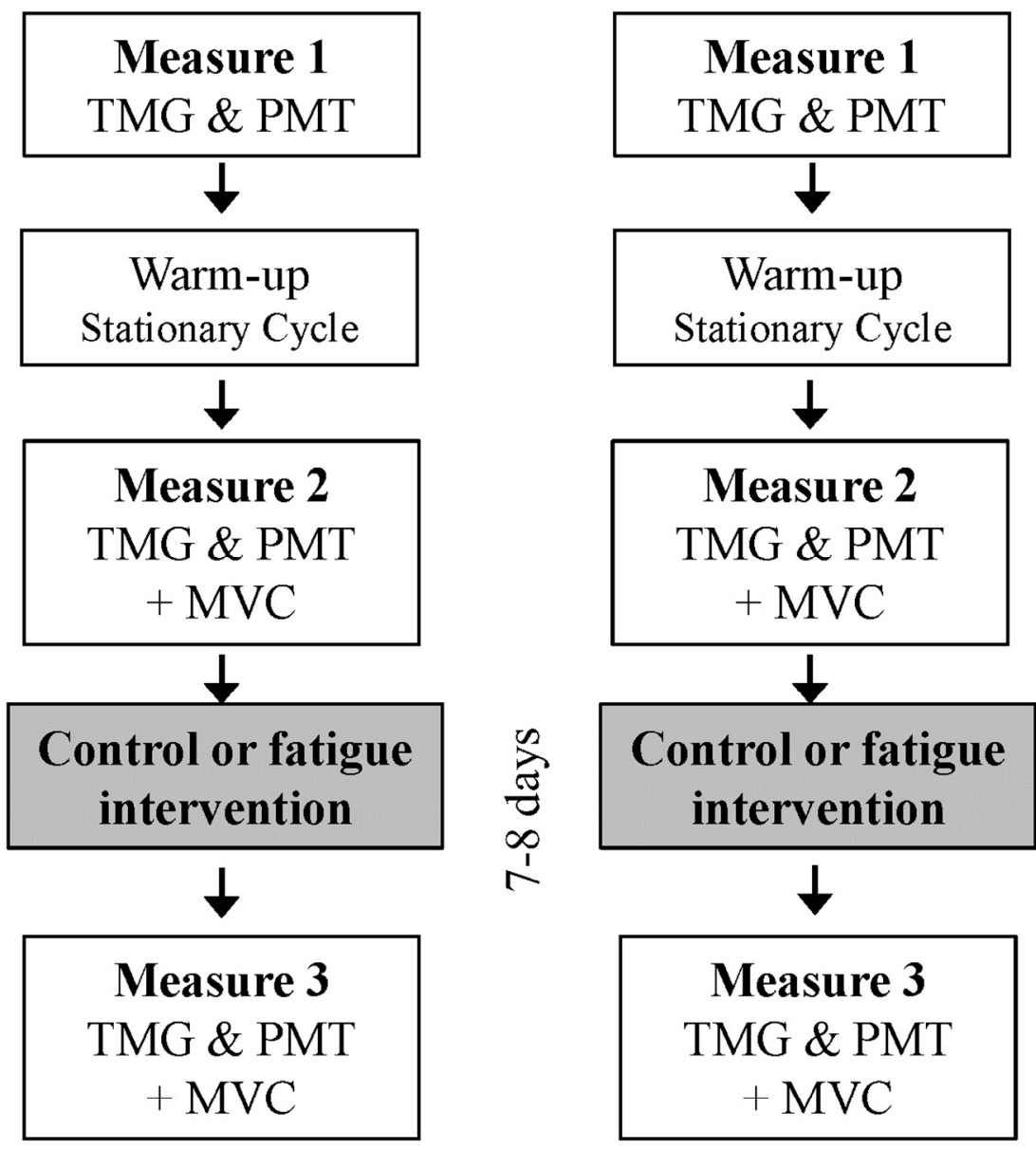

Figure 1. Schematic representation of the research design. TMG = Tensiomyography; PMT = passive muscle tension.

$120 \times 150 \mathrm{~mm}(300 \times 300 \mathrm{DPI})$ 


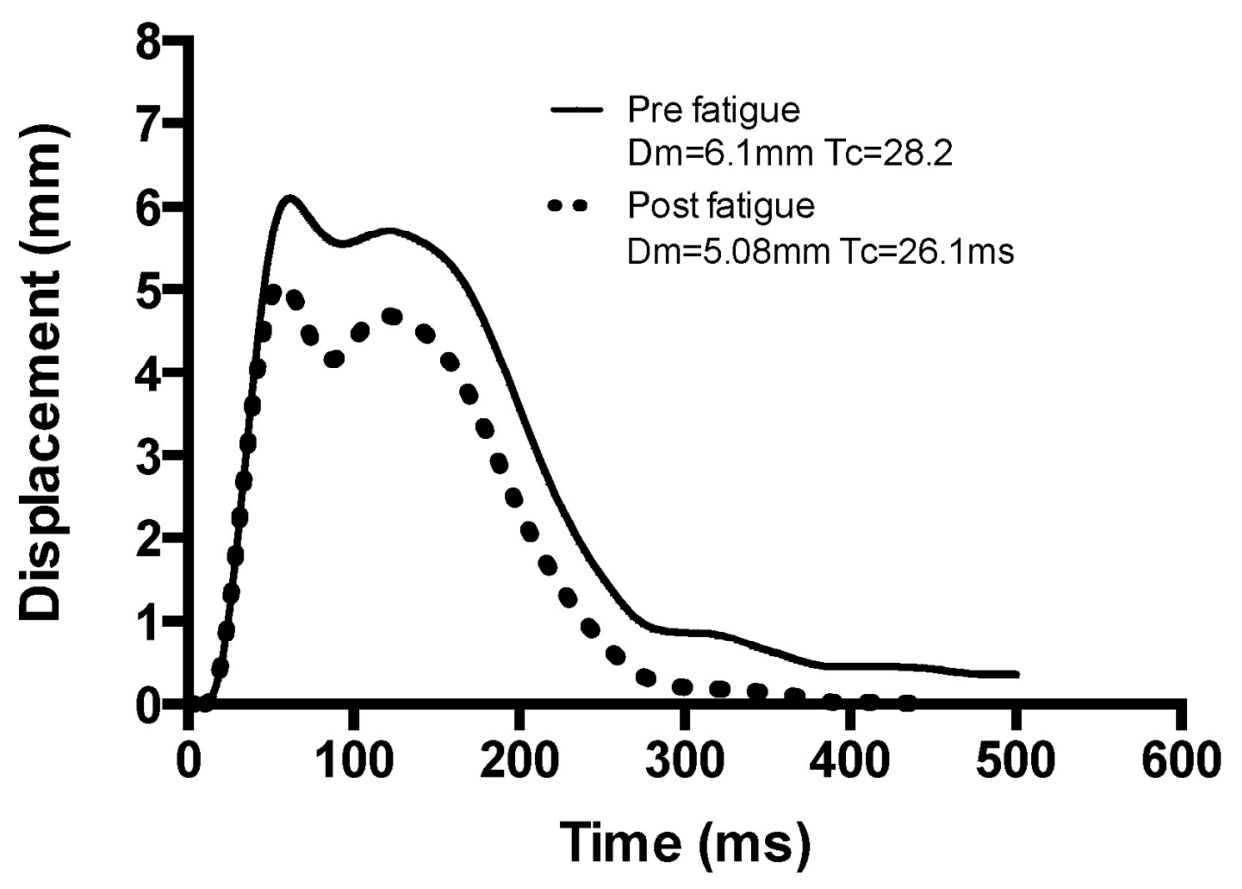

Figure 2. Typical displacement/ time curve of the tensiomyographic signal before and after the administration of the fatigue protocol. $\mathrm{Dm}=$ muscle displacement; $\mathrm{Tc}=$ contraction time . $150 \times 110 \mathrm{~mm}(300 \times 300 \mathrm{DPI})$ 


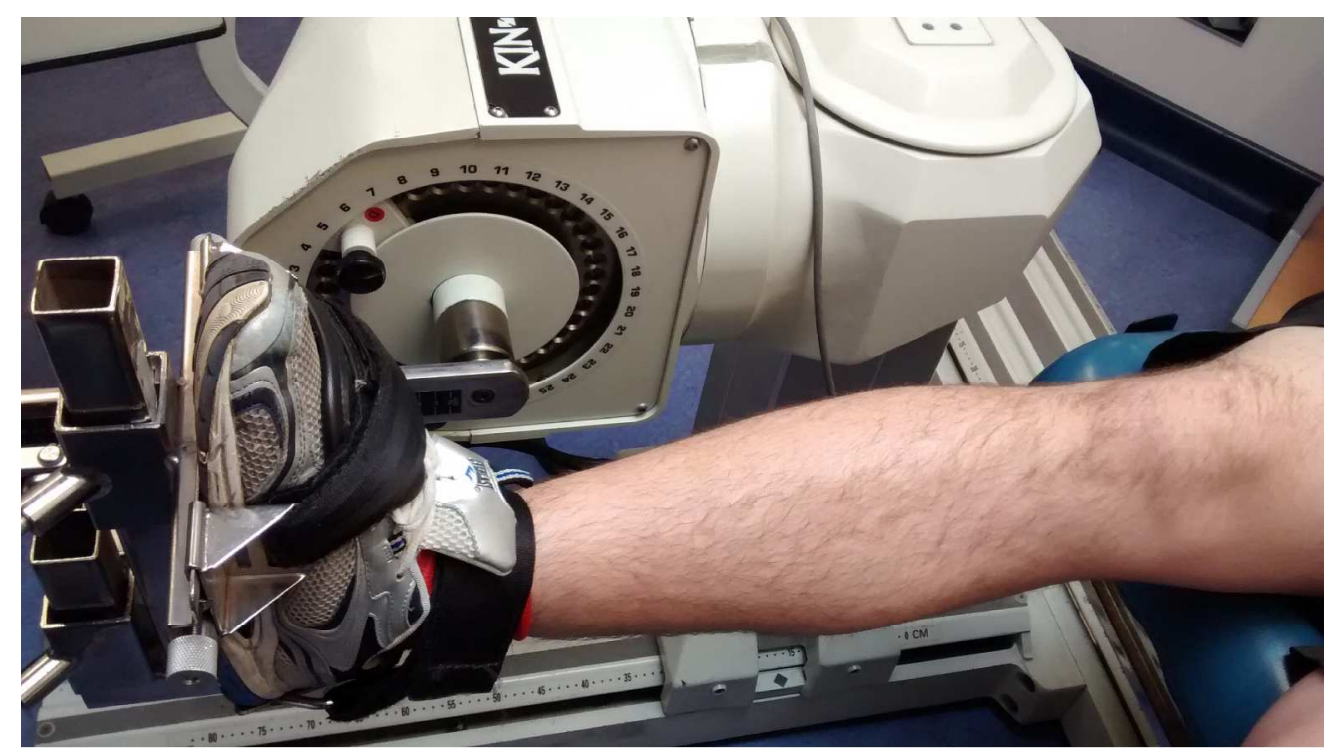

Figure 3. Isokinetic dynamometer setup for PMT and MVC assessment. Ankle flexed at $90^{\circ}$ relative to the tibia.

$342 \times 192 \mathrm{~mm}$ (300 x 300 DPI) 


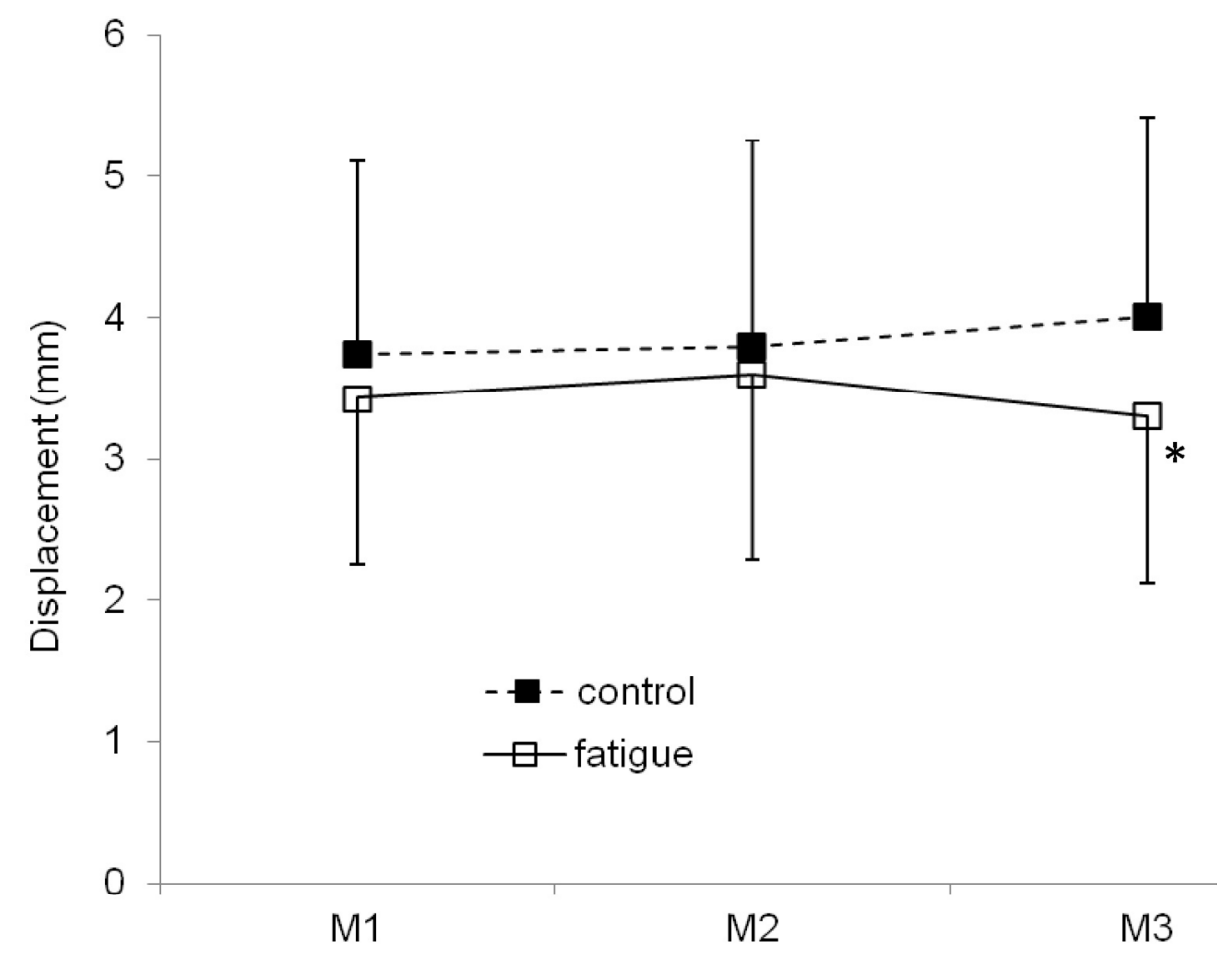

Figure 4. Average values (+ SD) of passive muscle tension as assessed on the isokinetic dynamometer at the three measurement points. ${ }^{*}=$ significant different from 'control' at M3, $\mathrm{p}<0.01$. $442 \times 383 \mathrm{~mm}$ (96 x 96 DPI) 


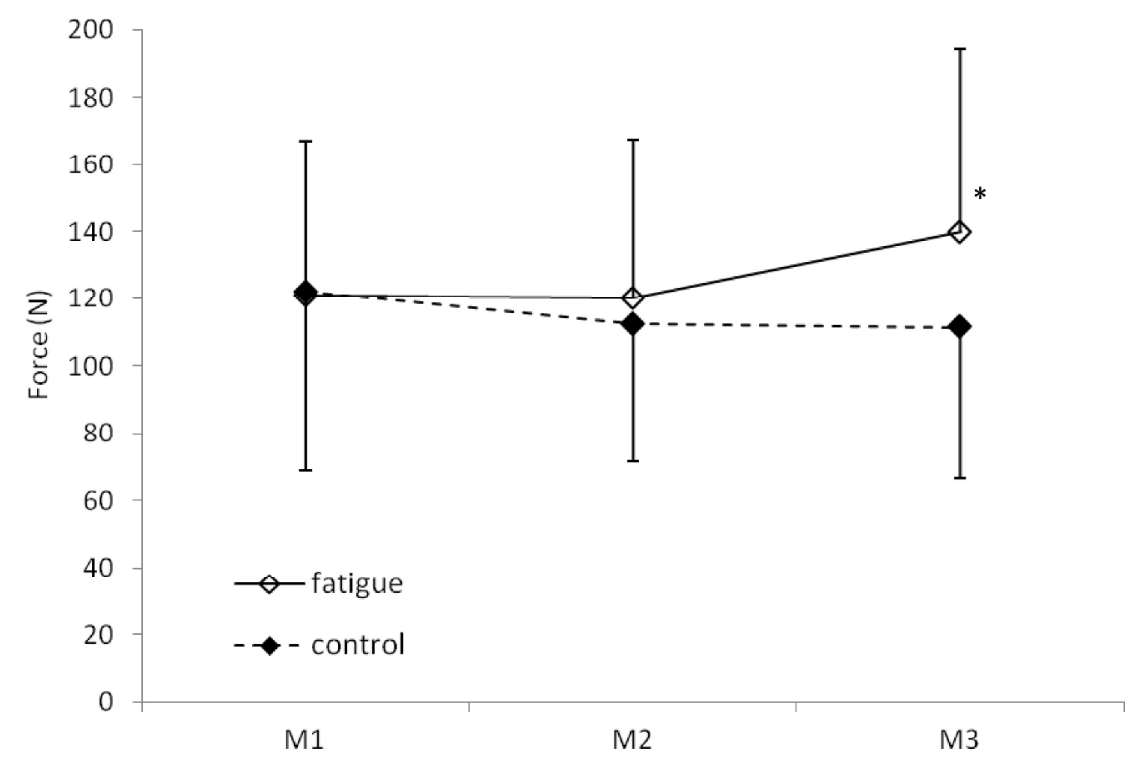

Figure 5. Average values (+ SD) of muscle displacement as assessed by tensiomyography at the three measurement points. ${ }^{*}=$ significant different from 'control' at M3, $\mathrm{p}<0.05$.

$254 \times 190 \mathrm{~mm}(300 \times 300 \mathrm{DPI})$ 